\title{
ON CERTAIN APPLICATIONS OF THE KHUKHRO-MAKARENKO THEOREM
}

\author{
AHMET ARIKAN \\ Gazi Üniversitesi, Gazi Eğitim Fakültesi, Matematik Eğitimi Anabilim Dalı 06500 Teknikokullar, \\ Ankara, Turkey \\ e-mail:arikan@gazi.edu.tr \\ HOWARD SMITH \\ Department of Mathematics, Bucknell University, Lewisburg, PA 17837, USA \\ e-mail: howsmith@bucknell.edu \\ and NADIR TRABELSI \\ Laboratory of Fundamental and Numerical Mathematics, Department of Mathematics, \\ University Ferhat Abbas of Setif, Algeria \\ e-mail: nadir_trabelsi@yahoo.fr
}

(Received 23 December 2011; revised 7 May 2012; accepted 9 May 2012; first published online 2 August 2012)

\begin{abstract}
Some recent results of Khukhro and Makarenko on the existence of characteristic $\mathfrak{X}$-subgroups of finite index in a group $G$, for certain varieties $\mathfrak{X}$, are used to obtain generalisations of some well-known results in the literature pertaining to groups $G$, in which all proper subgroups satisfy some condition or other related to the property 'soluble-by-finite'. In addition, a partial generalisation is obtained for the aforementioned results on the existence of characteristic subgroups.
\end{abstract}

2000 Mathematics Subject Classification. 20F10, 20F19, $20 \mathrm{E} 50$.

1. Introduction. Let $F$ be a free group of countable rank with basis $\left\{x_{1}, x_{2}, \ldots\right\}$. Then an outer commutator word of weight 1 is $x_{1}$, and an outer commutator word $\omega$ of weight $t>1$ is a word of the form

$$
\omega\left(x_{1}, \ldots, x_{t}\right)=\left[u\left(x_{1}, \ldots, x_{r}\right), v\left(x_{r+1}, \ldots, x_{t}\right)\right],
$$

where $u, v$ are outer commutator words of weight $r, t-r$ respectively. Let $\omega$ be an outer commutator word of weight $t$. We denote by $\mathfrak{X}_{\omega}$ the class of groups $G$ satisfying $\omega\left(g_{1}, \ldots, g_{t}\right)=1$ for all $g_{1}, \ldots, g_{t} \in G$, i.e. $\omega(G)=1$.

Some recent results of Khukhro and Makarenko (see especially Lemma 2.1) establish that, for certain group-theoretic properties $\mathfrak{Y}$, the existence of an $\mathfrak{Y}$-subgroup $H$ of finite index in a group $G$ ensures that there is a characteristic $\mathfrak{Y}$-subgroup $C$ of finite index in $G$. In the present paper we shall use these results to obtain generalisations of some well-known results on groups $G$, in which all proper subgroups satisfy certain conditions, in several cases the condition in question being either 'almost in the variety $\mathfrak{X}_{\omega}$ ' for some outer commutator word $\omega$ (see for example Theorem 2.4) or ' $\mathfrak{X}_{\omega}$-byChernikov' (see for example Theorem 2.5). We shall also obtain a generalisation of a result on barely transitive $p$-groups (see Theorem 2.3). Recall that a group 
of permutations $G$ of an infinite set $\Omega$ is called a barely transitive group if $G$ acts transitively on $\Omega$ and every orbit of every proper subgroup is finite. Equivalently, $G$ is barely transitive if $G$ has a subgroup $H$ such that $|G: H|$ is infinite, $\bigcap_{g \in G} H^{g}=1$ and $|K: K \cap H|$ is finite for every proper subgroup $K$ of $G$, where the subgroup $H$ is called a point stabiliser. Finally, in Section 4 of the paper, we obtain some partial generalisations of the Khukhro-Makarenko results.

We shall use the following notation for the given classes of groups.

$\mathfrak{A}$ : Abelian groups,

$\mathfrak{N}$ : Nilpotent groups,

S: Soluble groups,

$\mathfrak{S}_{d}$ : Soluble groups of derived length at most $d$,

$\mathfrak{C}$ : Chernikov groups,

$\mathfrak{R}$ : Groups of finite (Prüfer) rank,

$\mathfrak{F}$ : Finite groups,

$\mathfrak{D}$ : Divisible (radicable) groups,

$\mathfrak{T}$ : Periodic groups,

$\mathfrak{L}:(\mathfrak{T} \cap \mathfrak{D} \cap \mathfrak{A})$-groups.

We also denote the class of all $\mathfrak{X}$-by-YY-groups by $\mathfrak{X} Y$, and $\mathfrak{X} \mathfrak{X}$-groups by $\mathfrak{X}^{2}$.

2. $\mathfrak{X}_{\omega} \mathfrak{C}$-groups. We will use the following very useful result, referred to here as the Khukhro-Makarenko theorem.

Lemma 2.1 ([9, Theorem 1], [11, Theorem 1] or [13]). If a group G has a subgroup $H$ of finite index $n$ satisfying the identity $\chi(H)=1$, where $\chi$ is an outer commutator word of weight $w$, then $G$ has also a characteristic subgroup $C$ of finite $(n, w)$-bounded index satisfying the same identity $\chi(C)=1$.

Before we give an application of Lemma 2.1, we prove the following lemma.

LEMMA 2.2. Let $G$ be a group and let $\omega$ be an outer commutator word of weight $t \geq 2$; then $G^{(t-1)} \leq \omega(G)$. In particular,

(i) if $\omega(G)=1$, then $G$ is in $\mathfrak{S}_{t-1}$, i.e. $\mathfrak{X}_{\omega} \leq \mathfrak{S}_{t-1}$,

(ii) if $G$ is a perfect group, then $\omega(G)=G$.

Proof. We proceed by induction on $t$. If $t=2$, then $G^{(t-1)}=G^{(1)}=G^{\prime}=\omega(G)$. Now assume that $t \geq 3$; then there exist outer commutator words $\sigma, \tau$ of weight $1 \leq t_{1}, t_{2}<t$, respectively, such that $t=t_{1}+t_{2}$ and $\omega=[\sigma, \tau]$, and then $\omega(G)=[\sigma(G), \tau(G)]$. By induction hypothesis, we have $G^{\left(t_{1}-1\right)} \leq \sigma(G)$ and $G^{\left(t_{2}-1\right)} \leq \tau(G)$. Put $m=\max \left\{t_{1}, t_{2}\right\}$, then

$$
G^{(m)}=\left[G^{(m-1)}, G^{(m-1)}\right] \leq\left[G^{\left(t_{1}-1\right)}, G^{\left(t_{2}-1\right)}\right] \leq[\sigma(G), \tau(G)]=\omega(G) .
$$

Clearly $t_{1}+t_{2} \geq m+1$ and thus $t-1 \geq m$. So $G^{(t-1)} \leq G^{(m)} \leq \omega(G)$ and the induction is complete.

(i) If $\omega(G)=1$, then $G^{(t-1)}=1$. So $G$ is in $\mathfrak{S}_{t-1}$.

(ii) Assume that $G$ is a perfect group. Since $G^{(t-1)} \leq \omega(G)$, we have $G^{(t-1)}=G$, and hence $G=\omega(G)$, as desired.

As an application of the Khukhro-Makarenko theorem we present the following result. 
THEOREM 2.3. Let $G$ be a locally finite barely transitive p-group with a point stabiliser $H$ and let $\omega$ be an outer commutator word of weight $t$. If $H \in \mathfrak{X}_{\omega}$, then $G^{\prime} \neq G$ and $G^{\prime} \in \mathfrak{X}_{\omega}$.

Proof. Let $N$ be a proper normal subgroup of $G$; then $N \cap H \in \mathfrak{X}_{\omega}$. Since $\mid N: N \cap$ $H \mid$ is finite, by Lemma $2.1, N$ has a characteristic subgroup $K \in \mathfrak{X}_{\omega}$ such that $N / K \in \mathfrak{F}$. It is well known that $G$ has no proper subgroup of finite index, so $N / K \leq Z(G / K)$. It follows that $N^{\prime} \leq K$ and that $N^{\prime} \in \mathfrak{X}_{\omega}$. Since there exists a chain $\left\{N_{i}: i \in I\right\}$ of proper normal subgroups of $G$ such that $G=\bigcup_{i \in I} N_{i}$, it follows that

$$
G^{\prime}=\bigcup_{i \in I} N_{i}^{\prime} .
$$

Consequently, we have $G^{\prime} \in \mathfrak{X}_{\omega}$ and $G \neq G^{\prime}$ by Lemma 2.2(ii).

Theorem 2.3 generalises [1] and [2, Theorem 2], and by using Lemma 2.2(i) we can obtain the same results as those in [1] and [2, Theorem 2]. The structure of imperfect locally finite barely transitive groups is described in [7].

Let $v\left(x_{1}, \ldots, x_{s}\right)$ and $u\left(x_{1}, \ldots, x_{t}\right)$ be two words. Then the composite of $v$ and $u$, $v \circ u$ is defined as follows:

$$
v \circ u=v\left(u\left(x_{1}, \ldots, x_{t}\right), \ldots, u\left(x_{(s-1) t+1}, \ldots, x_{s t}\right)\right) .
$$

If $v$ is an outer commutator word and $u$ is a word, then it is well known that $v \circ u(G)=$ $v(u(G))$ for any group $G$ (see for example [16, Lemma 2.5]).

We will use this definition to describe the structure of certain groups.

Let $\mathfrak{Y}$ be a class of groups. Recall that a group $G$ is called a minimal non- $\mathfrak{Y}$ group if every proper subgroup of $G$ is a $\mathfrak{Y}$-group, but $G$ itself is not. The minimal non-Y)-groups are denoted by $M N \mathfrak{Y}$.

Now define the word $\theta$ as $\theta(x, y)=[x, y]$, which will be used in the sequel.

THEOREM 2.4. Let $G$ be an $M N \mathfrak{X}_{\omega} \mathfrak{F}_{\text {-group, }}$ where $\omega$ is an outer commutator word of weight $t>1$. If G has no infinite simple images, then the following properties hold.

(i) $G$ has no proper subgroup of finite index and no simple images.

(ii) $N^{\prime} \in \mathfrak{X}_{\omega}$ for every proper normal subgroup $N$ of $G$.

(iii) $G$ is not perfect, $G \in \mathfrak{X}_{\omega}(\mathfrak{L} \cap \mathfrak{C})$ and $G^{\prime} \in \mathfrak{X}_{\omega}$. In particular, $G \in \mathfrak{S}_{t}$.

(iv) $(\omega \circ \theta)(G)=1$, i.e. $G \in \mathfrak{X}_{\omega \circ \theta}$.

Proof. We first assume that $G$ has a proper subgroup $K$ of finite index. Since $K \in \mathfrak{X}_{\omega} \mathfrak{F}, K$ has a normal subgroup $L \in \mathfrak{X}_{\omega}$ such that $K / L \in \mathfrak{F}$. Hence, core $_{G} L \in \mathfrak{X}_{\omega}$ and has finite index in $G$, i.e. $G \in \mathfrak{X}_{\omega} \mathfrak{F}$. But this is a contradiction. So $G$ has no proper subgroup of finite index and it has no simple images. Thus (i) holds.

Now let $N$ be a proper normal subgroup of $G$. Since $N \in \mathfrak{X}_{\omega} \mathfrak{F}, N$ has a characteristic subgroup $S \in \mathfrak{X}_{\omega}$ of finite index in $N$ by Lemma 2.1. Put $\bar{G}:=G / S$, then $\bar{G}=C_{\bar{G}}(\bar{N})$, since $G / S$ has no proper subgroup of finite index and so we have $[G, N] \leq S$. Since $\mathfrak{X}_{\omega}$ is subgroup-closed, $N^{\prime} \in \mathfrak{X}_{\omega}$, and thus (ii) holds.

Now assume that $G$ is perfect. Since $G$ has no simple images, it is a union of a chain of proper normal subgroups. If $N$ is a proper normal subgroup of $G$, then $N^{\prime} \in \mathfrak{X}_{\omega}$ by (ii) and so $G=G^{\prime}$ is a union of $\mathfrak{X}_{\omega}$-groups. So $G \in \mathfrak{X}_{\omega}$, a contradiction.

Thus, $G$ is not perfect and $G / G^{\prime}$ has a proper subgroup $R / G^{\prime}$ such that $G / R \in \mathfrak{L} \cap \mathfrak{C}$. Now by Lemma $2.1, R$ has a characteristic subgroup $W \in \mathfrak{X}_{\omega}$ such that $G / W \in \mathfrak{C}$. Since $G / W$ has no proper subgroup of finite index, we have $G / W \in \mathfrak{L} \cap \mathfrak{C}$. 
Consequently, $G \in \mathfrak{X}_{\omega}(\mathfrak{L} \cap \mathfrak{C})$. In particular, $G^{\prime} \leq W$ and hence $G^{\prime} \in \mathfrak{X}_{\omega}$. In particular, $G \in \mathfrak{S}_{t}$ by Lemma 2.2(i). So (iii) holds.

Finally, since $G^{\prime} \in \mathfrak{X}_{\omega}$, we have $(\omega \circ \theta)(G)=\omega\left(G^{\prime}\right)=1$, and (iv) holds.

The following is the $\mathfrak{X}_{\omega} \mathfrak{C}$ version of Theorem 2.4.

THEOREM 2.5. Let $G$ be an $M N \mathfrak{X}_{\omega} \mathfrak{C}$-group. If $G$ has no infinite simple images, then the following are satisfied.

(i) G has no proper subgroup of finite index and no simple images.

(ii) $N^{\prime} \in \mathfrak{X}_{\omega \circ \theta}$ for every proper normal subgroup $N$ of $G$, i.e. $N \in \mathfrak{X}_{\omega \circ \theta^{2}}$.

(iii) $G$ is not perfect and $G \in \mathfrak{X}_{\omega \circ \theta}(\mathfrak{L} \cap \mathfrak{C})$. In particular, $G^{\prime} \in \mathfrak{X}_{\omega \circ \theta}$ and $G \in \mathfrak{S}_{t+1}$.

Proof. By a similar argument to that used in the proof of Theorem 2.4, $G$ has no proper subgroup of finite index. So (i) holds.

Now let $N$ be a proper normal subgroup of $G$, then it has a normal subgroup $S \in \mathfrak{X}_{\omega}$ such that $N / S \in \mathfrak{C}$. So $N / S$ has a normal subgroup $R / S \in \mathfrak{L} \cap \mathfrak{C}$ such that $N / R \in \mathfrak{F}$. Since $R / S$ is in $\mathfrak{A}, R \in \mathfrak{X}_{\omega \circ \theta}$. By Lemma $2.1 N$ has a characteristic subgroup $M \in \mathfrak{X}_{\omega \circ \theta}$ such that $N / M \in \mathfrak{F}$ and hence $N^{\prime} \leq M$, i.e. $N^{\prime} \in \mathfrak{X}_{\omega \circ \theta}$. So (ii) holds.

Suppose next that $G$ has a non-trivial $\mathfrak{C}$-image $G / N$. Then $N$ has a normal subgroup $S \in \mathfrak{X}_{\omega}$ such that $N / S \in \mathfrak{C}$ and $N / S$ has a normal subgroup $M / S \in \mathfrak{L} \cap \mathfrak{C}$ such that $N / M \in \mathfrak{F}$. So $N \in \mathfrak{X}_{\omega \circ \theta} \mathfrak{F}$. By Lemma $2.1 N$ has a characteristic subgroup $T \in \mathfrak{X}_{\omega \circ \theta}$ with $N / T \in \mathfrak{F}$. This implies that $G / T \in \mathfrak{C}$, and hence $G / T \in \mathfrak{L} \cap \mathfrak{C}$ by (i) and $G \in$ $\mathfrak{X}_{\omega \circ \theta}(\mathfrak{L} \cap \mathfrak{C})$ in this case.

Now if $G$ is perfect, then as in the proof of Theorem 2.4, $G$ is a union of proper normal subgroups and so we have $G \in \mathfrak{X}_{\omega \circ \theta^{2}}$, and hence $\omega(G)=1$, a contradiction. So $G$ is not perfect and $G / G^{\prime}$ has a proper normal subgroup $R / G^{\prime}$ such that $G / R \in$ $\mathfrak{L} \cap \mathfrak{C}$. By the previous argument $G \in \mathfrak{X}_{\omega \circ \theta}(\mathfrak{L} \cap \mathfrak{C})$ and so $G^{\prime} \in \mathfrak{X}_{\omega \circ \theta}$ and $G \in \mathfrak{S}_{t+1}$ by Lemma 2.2(i). Thus, (iii) holds.

3. Applications to $M N \mathfrak{S}_{n} \mathfrak{C}$ and $M N \mathfrak{S}_{n} \mathfrak{F}$-groups. Since a group is in $\mathfrak{S C}$ if and only if it is in $\mathfrak{S F}$, we see that a group is in $M N \mathfrak{S C}$ if and only if it is in $M N \mathfrak{S F}$.

We know that the celebrated example of Heineken and Mohamed (see [15, Theorem 6.2.1]) is an $M N \mathfrak{A F}$-group which is in $\mathfrak{A C}$. So an $M N \mathfrak{S}_{n} \mathfrak{F}$-group (for a positive integer $n$ ) is not in general an $M N \mathfrak{S}_{n} \mathfrak{C}$-group.

The locally graded groups with all proper subgroups in $\mathfrak{S F}$ are classified by [6, Theorem C], as follows

THEOREM 3.1. Let $G$ be a locally graded group with all proper subgroups in $\mathfrak{S F}$. Then either

(i) $G$ is locally soluble, or

(ii) $G \in \mathfrak{S F}$, or

(iii) $G$ is $\mathfrak{S}-b y-P S L(2, \mathfrak{F})$, or

(iv) $G$ is $\mathfrak{S}-b y-S z(\mathfrak{F})$,

where $F$ is an infinite locally finite field with no infinite proper subfields.

By the remark above, we see that Theorem 3.1 also gives a classification of the locally graded groups with all proper subgroups in $\mathfrak{S C}$.

If $G$ is a countable locally graded simple group with all subgroups in $\mathfrak{S F}$ (or in $\mathfrak{S C}$ ), then a super-inert subgroup $R$ (see [6] for the definition) of $G$ either has non-trivial Hirsch-Plokin radical or is in $\mathfrak{F}$, hence $G$ is locally finite [6, Theorem 2]. So by [12] 
$G$ is isomorphic either to $\operatorname{PSL}(2, \mathbb{F})$ or to $S z(\mathbb{F})$ for some infinite locally finite field $\mathbb{F}$ containing no infinite proper subfield.

THEOREM 3.2. There are infinite locally finite simple $M N \mathfrak{S}_{2} \mathfrak{F}$ and $M N \mathfrak{S}_{3} \mathfrak{F}$-groups.

Proof. Let $G:=\operatorname{PSL}(2, \mathbb{F})$ or $G:=S z(\mathbb{F})$ for some infinite locally finite field $\mathbb{F}$ containing no infinite proper subfield. In the first case every proper subgroup is either in $\mathfrak{A}^{2}$ or in $\mathfrak{F}$ and so in $\mathfrak{S}_{2} \mathfrak{F}$ by [4, Example 3]. Clearly $G \notin \mathfrak{S}_{2} \mathfrak{F}$. So $G \in M N \mathfrak{S}_{2} \mathfrak{F}$.

In the second case every proper subgroup of $G$ is in $\mathfrak{F}$ or is $\mathfrak{N}_{2}$-by-locally cyclic (i.e. in $\mathfrak{S}_{3} \mathfrak{F}$ ) by the proof of [5, Lemma 2]. Consequently, $G$ is in $M N \mathfrak{S}_{3} \mathfrak{F}$.

Let $G$ be a group, $H$ a subgroup of $G$; then the isolator $I_{G}(H)$ of $H$ in $G$ is defined as

$$
I_{G}(H)=\left\{x \in G \mid \text { there is a non-zero integer } n \text { such that } x^{n} \in H\right\} .
$$

We prove the following general lemma.

LEMMA 3.3. Let $\omega$ be an outer commutator word of weight $t$, and let $H$ be a subgroup of the locally nilpotent torsion-free group $G$. Then

$$
\omega\left(I_{G}(H)\right) \leq I_{G}(\omega(H)) .
$$

Proof. First let $U$ and $V$ be subgroups of $G$. Then with the notation of [14, Section 2.3] we have $I_{G}(U) \sim U$ and $I_{G}(V) \sim V$. It follows that $\left[I_{G}(U), I_{G}(V)\right] \sim[U, V]$ by $[\mathbf{1 4}$, 2.3.5]. So we see that

$$
\left[I_{G}(U), I_{G}(V)\right] \leq I_{G}([U, V]
$$

Now we proceed by induction on $t$. If $t=1$, then the result is immediate. If $t>1$, then $\omega=[\varphi, \delta]$ for some outer commutator words $\varphi$ and $\delta$ of weights $1 \leq t_{1}<t$, $1 \leq t_{2}<t$ such that $t_{1}+t_{2}=t$. By induction hypothesis and the above remark, we have

$$
\begin{aligned}
\omega\left(I_{G}(H)\right)=\left[\varphi\left(I_{G}(H)\right), \delta\left(I_{G}(H)\right)\right] & \leq\left[I_{G}(\varphi(H)), I_{G}(\delta(H))\right] \\
& \leq I_{G}([\varphi(H), \delta(H)])=I_{G}(\omega(H)),
\end{aligned}
$$

and the proof is complete.

THEOREM 3.4. Let $G$ be a locally nilpotent torsion-free group.

(i) If all proper subgroups of $G$ are in $\mathfrak{X}_{\omega} \mathfrak{T}$, then $G \in \mathfrak{X}_{\omega}$.

(ii) If all proper subgroups of $G$ are in $\mathfrak{X}_{\omega} \mathfrak{R}$, then $G \in \mathfrak{X}_{\omega}(\mathfrak{R} \cap \mathfrak{N})$. In particular, $G$ is in $\mathfrak{S}$.

Proof. (i) Let $K$ be a proper subgroup of $G$. Then $K$ has a normal $\operatorname{subgroup} N \in \mathfrak{X}_{\omega}$ such that $K / N \in \mathfrak{T}$, and so $I_{K}(N)=K$. By Lemma 3.3

$$
\omega(K)=\omega\left(I_{K}(N)\right) \leq I_{K}(\omega(N))=I_{K}(1)=1 .
$$

This means that every proper subgroup $K$ of $G$ is in $\mathfrak{X}_{\omega}$.

If $G$ is not finitely generated, then every finitely generated subgroup of $G$ is in $\mathfrak{X}_{\omega}$, and thus $G \in \mathfrak{X}_{\omega}$. Otherwise, $G$ is finitely generated, and by $[18,5.2 .21]$ it has a normal 
subgroup $H \in \mathfrak{X}_{\omega}$ of finite index, since it is nilpotent. Hence, $I_{G}(H)=G$ and as above $\omega(G)=1$. So $G \in \mathfrak{X}_{\omega}$.

(ii) Assume for a contradiction that $G$ is not in $\mathfrak{X}_{\omega} \mathfrak{R}$, and first suppose that $G$ has a proper normal subgroup of $N$ such that $G / N$ is in $\Re$. Then $N$ has a normal subgroup $M$ such that $M \in \mathfrak{X}_{\omega}$ and $N / M \in \mathfrak{R}$. By [10, Theorem 3], we may assume that $M$ is characteristic in $N$ so that $M$ is normal in $G$. Clearly $G / M$ is in $\mathfrak{R}$, so $G \in \mathfrak{X}_{\omega} \mathfrak{R}$, a contradiction. Therefore, $G$ has no proper images which are in $\Re$ and hence it is perfect.

Let $H$ be any proper normal subgroup of $G$ and let $K$ be a characteristic subgroup of $H$ such that $K \in \mathfrak{X}_{\omega}$ and $H / K \in \mathfrak{R}$. If $T / K$ denotes the torsion subgroup of $H / K$, then $T \in \mathfrak{X}_{\omega} \mathfrak{T}$ and hence $T \in \mathfrak{X}_{\omega}$ by (i). Since torsion-free locally nilpotent $\mathfrak{R}$-groups are in $\mathfrak{N}$ [18, Theorem 6.36], we have $H / T \in \mathfrak{N}$. So $H / T$ has a finite characteristic series whose factors are torsion-free $\mathfrak{A} \cap \mathfrak{R}$-groups. If $U$ is such a factor, then $G / C_{G}(U)$ is nilpotent by [17, Part 2, Lemma 6.37] and hence $G=C_{G}(U)$ since $G$ is perfect. We deduce that $H / T$ is contained in the hypercentre of $G / T$, which equals the centre, as $G$ is perfect. Thus, $H / T \leq Z(G / T)$ and so $H^{\prime} \leq T$. We deduce that $H^{\prime} \in \mathfrak{X}_{\omega}$. As before, since there exists a chain $\left\{N_{i}: i \in I\right\}$ of proper normal subgroups of $G$ such that $G=\bigcup_{i \in I} N_{i}$, it follows that $G=G^{\prime}=\bigcup_{i \in I} N_{i}^{\prime}$. Consequently, we have $G \in \mathfrak{X}_{\omega}$, a contradiction. Therefore, $G \in \mathfrak{X}_{\omega} \mathfrak{R}$.

Let $N$ be a normal subgroup of $G$ such that $N \in \mathfrak{X}_{\omega}$ and $G / N \in \mathfrak{R}$. If $T / N$ denotes the torsion subgroup of $G / N$, then again by (i) $T \in \mathfrak{X}_{\omega}$, and since $G / T$ is a locally nilpotent torsion-free $\mathfrak{R}$-group, it is in $\mathfrak{N}$. Therefore, $G \in \mathfrak{X}_{\omega}(\mathfrak{R} \cap \mathfrak{N})$. By Lemma 2.2(i), we deduce that $G$ is in $\mathfrak{S}$, as claimed.

Let us define the outer commutator word $\phi_{j}$ for every $j \geq 0$ as follows:

$\phi_{0}(x)=x$ and for $i \geq 1$

$$
\phi_{i}\left(x_{1}, \ldots, x_{2^{i}}\right)=\left[\phi_{i-1}\left(x_{1}, \ldots, x_{2^{i-1}}\right), \phi_{i-1}\left(x_{2^{i-1}+1}, \ldots, x_{2^{i}}\right)\right] .
$$

Then $G$ is in $\mathfrak{S}$ if and only if there is a positive integer $n$ such that $\phi_{n}(G)=1$.

THEOREM 3.5. Let $G$ be a group without infinite simple images. Then the following are satisfied.

(i) If every proper subgroup of $G$ is in $\mathfrak{S}_{n} \mathfrak{F}$ for some fixed positive integer $n$, then either $G \in \mathfrak{S}_{n} \mathfrak{F}$ or $G \in \mathfrak{S}_{n}(\mathfrak{L} \cap \mathfrak{C})$. So if $G$ is an $M N \mathfrak{S}_{n} \mathfrak{F}$-group, then $G \in \mathfrak{S}_{n} \mathfrak{C} \cap \mathfrak{S}_{n+1}$.

(ii) If every proper subgroup of $G$ is in $\mathfrak{S}_{n} \mathfrak{C}$ for some fixed positive integer $n$, then $G \in$ $\mathfrak{S}_{n} \mathfrak{C}$ or $G \in \mathfrak{S}_{n+1}(\mathfrak{L} \cap \mathfrak{C})$. So if $G$ is an $M N \mathfrak{S}_{n} \mathfrak{C}$-group, then $G \in \mathfrak{S}_{n+1} \mathfrak{C} \cap \mathfrak{S}_{n+2}$.

Proof. (i) Take $\omega=\phi_{n}$. If $G \notin \mathfrak{S}_{n} \mathfrak{F}$, then $G$ is an $M N \mathfrak{S}_{n} \mathfrak{F}$-group. By Theorem 2.4 (iii) $G \in \mathfrak{X}_{\phi_{n}}(\mathfrak{L} \cap \mathfrak{C})$.

(ii) Again take $\omega=\phi_{n}$ so that $\omega \circ \theta=\phi_{n+1}$. If $G \notin \mathfrak{S}_{n} \mathfrak{C}$, then by Theorem 2.5 (iii) $G \in \mathfrak{X}_{\phi_{n+1}}(\mathfrak{L} \cap \mathfrak{C})$, and the proof is complete.

The following lemma will be generalised in Section 4 (see Lemma 4.1), but since the 'soluble' version of the lemma is useful here, we shall prove it.

Lemma 3.6 (c.f. [3, Proposition 1]). Let $G$ be in $\mathfrak{T} \cap \mathfrak{N}, A \in \mathfrak{S}_{n}(n \geq 1)$ a normal subgroup of $G$ such that $G / A \in \mathfrak{L}$. Then also $G \in \mathfrak{S}_{n}$.

Proof. We proceed by induction on $n$. If $n=1$, then $A$ is in $\mathfrak{A}$ and hence $A \leq$ $C_{G}(A)$. So $T:=G / C_{G}(A) \in \mathfrak{L}$ is isomorphic to a subgroup of Aut $A$. By [3, Lemma 1], $A \leq Z(G)$, and by [15, Section 5.3.5] $G$ is in $\mathfrak{A}$. Now let $n>1$ and consider $G / A^{(n-1)}$. 
Then

$$
\frac{G / A^{(n-1)}}{A / A^{(n-1)}} \in \mathfrak{L} \text { and } A / A^{(n-1)} \in \mathfrak{S}_{n-1} .
$$

By induction hypothesis $G / A^{(n-1)} \in \mathfrak{S}_{n-1}$ and thus $G^{(n-1)}=A^{(n-1)}$. This implies that $G^{(n)}=1$ and $G \in \mathfrak{S}_{n}$, as desired.

THEOREM 3.7. Let $G$ be a locally graded $\mathfrak{T}$-group and suppose that every proper subgroup of $G$ is in $\mathfrak{S}_{n} \mathfrak{C}$ for some fixed positive integer $n$. If $G$ contains a normal $\mathfrak{N}$ subgroup $N$ such that $G / N \in \mathfrak{C}$, then $G \in \mathfrak{S}_{n} \mathfrak{C}$.

Proof. Assume for a contradiction that $G$ is an $M N \mathfrak{S}_{n} \mathfrak{C}$-group. Since $G$ has no proper subgroup of finite index, we have $G / N \in \mathfrak{L}$ or $G=N$. Hence, $G^{\prime} \neq G$ and thus $1 \neq G / G^{\prime} \in \mathfrak{L}$. If $N=G$, then we have the contradiction that $G$ is in $\mathfrak{A}$ by $[\mathbf{1 8}$, Section 5.2.5], since $G$ is in $\mathfrak{T} \cap \mathfrak{N}$. So $N \neq G$ and hence $N$ has a normal subgroup $S \in \mathfrak{S}_{n}$ such that $N / S \in \mathfrak{C}$. So $N / S$ contains a maximal $\mathfrak{L}$-subgroup $R / S$ such that $N / R \in \mathfrak{F}$. By Lemma 3.6, $R \in \mathfrak{S}_{n}$. Therefore, we can assume by Lemma 2.1 that $R$ is characteristic in $N$ and hence $R$ is normal in $G$. So $G / R$ is in $\mathfrak{C}$. Consequently, $G \in \mathfrak{S}_{n} \mathfrak{C}$, a contradiction, and the proof is complete.

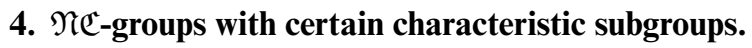

LEMMA 4.1. Let $G$ be in $\mathfrak{T} \cap \mathfrak{N}, N$ a normal subgroup of $G, \omega$ an outer commutator word of weight $t \geq 2$ such that $\omega(N)=1$. If $G / N$ is in $\mathfrak{A} \cap \mathfrak{D}$, then $\omega(G)=1$.

Proof. We proceed by induction on $t$. If $t=2$, then $\omega(x, y)=[x, y]$ and

$$
\omega(N)=[N, N]=1,
$$

i.e. $N$ is in $\mathfrak{A}$. So $G / C_{G}(N)$ is in $\mathfrak{A} \cap \mathfrak{D}$ and isomorphic to a subgroup of Aut $N$. By [3, Lemma 1] $G=C_{G}(N)$ and thus $N \leq Z(G)$. Applying [18, 5.3.5] we have that $G$ is in $\mathfrak{A}$. Let $t>2$; then $\omega=[\psi, \phi]$ for some outer commutator words $\psi, \phi$ of weight $1 \leq t_{1}, t_{2}<t$ such that $t=t_{1}+t_{2}$. Now $G / N$ is in $\mathfrak{L}$ and $\psi(N / \psi(N))=1$. If $t_{1}>1$, then by induction hypothesis $\psi(G / \psi(N))=1$, i.e. $\psi(G) \leq \psi(N)$. Clearly $\psi(N) \leq \psi(G)$ and it follows that $\psi(G)=\psi(N)$. If also $t_{2}>1$, then similarly $\phi(G)=\phi(N)$, and we have

$$
\omega(G)=[\psi(G), \phi(G)]=[\psi(N), \phi(N)]=1,
$$

as required. So we may assume that $t_{2}=1$ and hence $t_{1}>1$, since $t>2$. (If $t_{1}=1$, then a similar argument works.) Then $\omega(N)=[\psi(N), N]=1$ and hence $N \leq C_{G}(\psi(N))$. We also have that $\psi(N)$ is in $\mathfrak{A}$. Then $G / C_{G}(\psi(N))$ is in $\mathfrak{A} \cap \mathfrak{D}$ and isomorphic to a subgroup of Aut $\psi(N)$. So by [3, Lemma 1] $\psi(N) \leq Z(G)$; in other words $[\psi(N), G]=1$. It follows that

$$
\omega(G)=[\psi(G), G]=[\psi(N), G]=1,
$$

and the proof is complete.

THEOREM 4.2. Let $G$ be a $\mathfrak{T}$-group and let $N \in \mathfrak{N}_{c} \cap \mathfrak{X}_{\omega}$ be a normal subgroup of $G$ such that $G / N \in \mathfrak{C}$ for some outer commutator word $\omega$. Then $G$ contains a characteristic 
(even invariant under all surjective endomorphisms) subgroup $S \in \mathfrak{N}_{c} \cap \mathfrak{X}_{\omega}$ such that $G / S \in \mathfrak{C}$.

Proof. Let $W:=\left\langle N^{\alpha}\right| \alpha \in$ Aut $\left.G\right\rangle$, then $W$ is characteristic in $G$ and $W / N \in \mathfrak{C}$. By [8, Lemma 4.7] $W$ is in $\mathfrak{N}$. We also have that $W / N$ has a normal $\mathfrak{A} \cap \mathfrak{D}$-subgroup $R / N \in \mathfrak{R}$ such that $W / R$ is in $\mathfrak{F}$. Now by Lemma 4.1 we have $R \in \mathfrak{N}_{c} \cap \mathfrak{X}_{\omega}$. By Lemma 2.1 $W$ has characteristic (even invariant under all surjective endomorphisms) subgroups $S_{1} \in \mathfrak{N}_{c}$ and $S_{2} \in \mathfrak{X}_{\omega}$ such that $W / S_{i}$ is in $\mathfrak{F}$ for $i=1,2$. Put $S=S_{1} \cap S_{2}$, then $|W: S|<\infty$ and $S$ is contained in $\mathfrak{N}_{c} \cap \mathfrak{X}_{\omega}$. Since $W$ is characteristic in $G$, we see that $S$ is characteristic in $G$, and since $G / W \in \mathfrak{C}$ and $W / S$ is finite, we have $G / S \in \mathfrak{C}$. The proof is complete.

If we take $\omega=\gamma_{c+1}$, then

$$
\mathfrak{N}_{c} \cap \mathfrak{X}_{\omega}=\mathfrak{N}_{c} \cap \mathfrak{N}_{c}=\mathfrak{N}_{c} .
$$

Hence, we obtain the following result.

COROLlARY 4.3. Let $G$ be a $\mathfrak{T}$-group and let $N \in \mathfrak{N}_{c}$ be a normal subgroup of $G$ such that $G / N \in \mathfrak{C}$. Then $G$ contains a characteristic (even invariant under all surjective endomorphisms) subgroup $S \in \mathfrak{N}_{c}$ such that $G / S \in \mathfrak{C}$.

Corollary 4.3 sharpens [8, Lemma 4.7] and generalises [3, Lemma 3] and [9, Corollary 1(i)] in the periodic case.

In [8, p. 321] Hartley gives an example that shows that the 'periodicity' condition cannot be removed from the hypothesis of Corollary 4.3 and defined Chernikovsubnormality ( $\mathfrak{C}$-subnormality, in short) as follows:

A subgroup $N$ of a group $G$ is called $\mathfrak{C}$-subnormal in $G$ if there is a finite series

$$
N=N_{0} \unlhd N_{1} \unlhd \cdots \unlhd N_{r}=G
$$

such that $N_{i+1} / N_{i} \in \mathfrak{C}$ for $0 \leq i \leq r-1$.

COROLlaRY 4.4. Let $G$ be a $\mathfrak{T}$-group containing a $\mathfrak{C}$-subnormal subgroup $N \in \mathfrak{N}_{c}$. Then $G$ contains a characteristic (even invariant under all surjective endomorphisms) subgroup $S \in \mathfrak{N}_{c}$ such that $G / S \in \mathfrak{C}$.

Proof. The result follows by Corollary 4.3 and a simple induction.

We can give an immediate application of Corollary 4.3 by considering the following result due to Hartley.

THEOREM 4.5 [8, Theorem B]. If $G$ is a locally finite group admitting an involutory automorphism $\phi$ such that $C_{G}(\phi)$ is in $\mathfrak{C}$, then both $[G, \phi]^{\prime}$ and $G /[G, \phi]$ are in $\mathfrak{C}$.

As Shumyatsky mentions in [19, p. 160], if we take $N=C_{[G, \phi]}\left([G, \phi]^{\prime}\right)$, then $N \in \mathfrak{N}_{2}$, $G / N \in \mathfrak{C}$ and $N$ is $\phi$-invariant. So by Corollary $4.3, G$ has a characteristic subgroup $S \in \mathfrak{N}_{2}$ such that $G / S \in \mathfrak{C}$.

We record here the following theorem, which is an immediate consequence of Lemma 2.1.

THEOREM 4.6. Let $G$ be a group and let $N \in \mathfrak{X}_{\omega}$ be a normal subgroup of $G$ for some outer commutator word $\omega$ such that $G / N \in \mathfrak{C}$. Then $G$ contains a characteristic (even invariant under all surjective endomorphisms) subgroup $S \in \mathfrak{X}_{\omega \circ \theta}$ such that $G / S$ is finite. 
Proof. Since $G / N \in \mathfrak{C}$, there exists a normal $\mathfrak{A} \cap \mathfrak{D}$-subgroup $R / N$ of $G / N$ such that $G / R$ is in $\mathfrak{F}$. Since $N \in \mathfrak{X}_{\omega}$ and $R / N$ is in $\mathfrak{A}$, we have $R \in \mathfrak{X}_{\omega \circ \theta}$. By Lemma 2.1 $G$ has a characteristic subgroup (even invariant under all surjective endomorphisms) $S \in \mathfrak{X}_{\omega \circ \theta}$ such that $G / S$ is in $\mathfrak{F}$, and the result is established.

Of course, if we replace the condition $G / N \in \mathfrak{C}$ with $G / N \in \mathfrak{A F}$ in Theorem 4.6, then the result remains true.

\section{REFERENCES}

1. A. Arıkan, On barely transitive $p$-groups with soluble point stabilizer, J. Group Theory 5 (2002), 441-442.

2. V. V. Belyaev and M. Kuzucuoglu, Locally finite barely transitive groups, Algebra Logic 42 (2003), 147-152 (translated from Algebra i Logika 42 (2003), 261-270).

3. B. Bruno and F. Napolitani, A note on nilpotent-by-Cernikov groups, Glasgow. Math. J. 46 (2004), 211-215.

4. M. R. Dixon, M. J. Evans and H. Smith, Locally (soluble-by-finite) groups with all proper non-nilpotent subgroups of finite rank, J. Pure Appl. Algebra 135 (1999), 33-43.

5. M. R. Dixon, M. J. Evans and H. Smith, Groups with all proper subgroups nilpotentby-finite rank, Arch. Math. 75 (2000), 81-91.

6. M. R. Dixon, M. J. Evans and H. Smith, Groups with all proper subgroups soluble-byfinite rank, J. of Algebra $\mathbf{2 8 9}$ (2005), 135-147.

7. B. Hartley, On the normalizer condition and barely transitive permutation groups, Algebra Logic 13 (1974), 334-340 (translated from Russian from Algebra i Logika 13 (1974), 589-602).

8. B. Hartley, Periodic locally soluble groups containing an element of prime order with Chernikov centralizer, Quart. J. Math. Oxford Ser. 42(2) (1982), 309-323.

9. E. I. Khukhro and N. Yu. Makarenko, Large characteristic subgroups satisfying multilinear commutator identities, J. Lond. Math. Soc. 75(2) (2007), 635-646.

10. E. I. Khukhro and N. Yu. Makarenko, Automorphically invariant ideals satisfying multilinear identities, and group-theoretic applications, J. Algebra 320 (2008), 1723-1740.

11. E. I. Khukhro, A. A. Klyachko, N. Yu. Makarenko and Yu. B. Mel'nikova, Automorphism invarience and identities, Bull. Lond. Math. Soc. 41 (2009), 804-816.

12. P. B. Kleidman and R. A. Wilson, A characterization of some locally finite simple groups of lie type, Arch. Mat. 48 (1987), 10-14.

13. A. A. Klyachko and Yu. B. Mel'nikova, A short proof of Makarenko-Khukhro Theorem on a large characteristic subgroups with identity, Sb. Math. 200 (2009), 661-664 (translated from Russian from Mat. Sb. 200 (2009), 33-36).

14. J. C. Lennox and D. J. S. Robinson, The theory of infinite soluble groups (Clarendon Press, Oxford, UK, 2004).

15. J. C. Lennox and S. E. Stonehewer, Subnormal subgroups of groups, Oxford Mathematical Monographs (Clarendon Press, Oxford, UK, 1987).

16. M. R. R. Moghaddam, On the Schur-Baer property, J. Austral. Math. Soc. A 31 (1981), $343-361$.

17. D. J. S. Robinson, Finiteness conditions and generalized soluble groups, Part 2 (SpringerVerlag, New York, 1972).

18. D. J. S. Robinson, A course in the theory of groups (Springer-Verlag, New York, 1982).

19. P. Shumyatsky, Centralizers in locally finite groups, Turk. J. Math. 31 (2007), 149-170. 